\title{
Knowledge elicitation techniques in a knowledge management context
}

\author{
Tatiana Gavrilova and Tatiana Andreeva
}

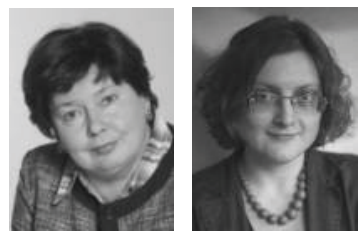

Tatiana Gavrilova is

Professor and Head of

Department in the

Department of Information

Technologies in

Management, St

Petersburg University

Graduate School of

Management, St

Petersburg, Russia.

Tatiana Andreeva is

Associate Professor in the

Department of

Organizational Behavior

and Human Resource

Management, St

Petersburg University

Graduate School of

Management, St

Petersburg, Russia.
Received: November 2011 Revised: January 2012 February 2012

Accepted: March 2012

\begin{abstract}
Purpose - A significant part of knowledge and experience in an organization belongs not to the organization itself, but to the individuals it employs. Therefore, knowledge management (KM) tasks should include eliciting knowledge from knowledgeable individuals. The paper aims to argue that the current palette of methods proposed for this in KM discourse is limited by idealistic assumptions about the behavior of knowledge owners. This paper also aims to enrich the repertoire of methods that can be used in an organization to extract knowledge (both tacit and explicit) from its employees by bridging KM and knowledge engineering and its accomplishments in the knowledge elicitation field.
\end{abstract}

Design/methodology/approach - This paper is based on extensive literature review and 20 years of experience of one of the authors in applying various knowledge elicitation techniques in multiple companies and contexts.

Findings - The paper proposes that the special agent (analyst) might be needed to elicit knowledge from individuals (experts) in order to allow further knowledge sharing and knowledge creation. Based on this idea, the paper proposes a new classification of the knowledge elicitation techniques that highlights the role of analyst in the knowledge elicitation process.

Practical implications - The paper contributes to managerial practice by describing a systemic variety of knowledge elicitation techniques with direct recommendations of their feasibility in the KM context.

Originality/value - The paper contributes to a wider use of knowledge engineering methodologies and technologies by KM researchers and practitioners in organizations.

Keywords Knowledge management, Knowledge elicitation techniques, Knowledge engineering

Paper type Conceptual paper

\section{Introduction}

Contemporary management theory views knowledge as one of the key sources for the creation and maintenance of a sustainable competitive advantage in a post-industrial economy (Kogut and Zander, 1992; Grant, 1996; Teece, 2004). Thus the question of how to ensure that organizations extract as much value as possible from their knowledge is topical from both conceptual and practical viewpoints.

However, knowledge and experience in an organization initially belong not to the organization itself, but to the individuals it employs (Grant, 1996; Tsoukas and Vladimirou, 2001). This fact, related to the very nature of knowledge, makes organizations dependent on both the good will and capabilities of employees in applying that knowledge for the organization's sake, and sharing it as needed by the organization. While some authors have discussed the transformation of individual into organizational knowledge (e.g. Tsoukas and Vladimirou, 2001; Nonaka, 1991), many sources remain pessimistic about the extent to which knowledge can be detached from an individual (Grant, 1996; Flood et al., 2001). So the question of what managers can do (if anything) to transform their employees' knowledge into organizational knowledge, or at least to share it as much as possible among various individuals in an organization, remains largely open. 
The aim of this study is to enhance the choices that can be made by organizations of how to extract knowledge (both tacit and explicit) from employees. The authors do this by bridging knowledge management (KM) and knowledge engineering (KE) and its accomplishments. $\mathrm{KE}$ is a discipline that also deals with managing knowledge, but from a different perspective, and it has a rich history of achievement over several decades (Burton et al., 1990; Cooke, 1994; Kendal and Creen, 2006). Surprisingly, although both deal with knowledge, KM and KE discourses have been developing in parallel and to the best of the authors' knowledge have rarely converged. In this paper the authors aim to bridge this gap and introduce some KE tools that can extend KM discourse; they propose a map that can guide the choice of tools.

To meet these goals, this paper is structured as follows. It starts with an overview of the existing knowledge management literature on the transformation of individual into organizational knowledge. Next, the authors briefly introduce knowledge engineering as a field of science and explain how it can help with the "bottlenecks" identified in the knowledge management literature. Then the authors review a number of knowledge elicitation methods developed within KE and discuss their advantages and disadvantages. The paper concludes with some implications for both knowledge management theory and practice, as well as with directions for future research.

\section{Knowledge of individuals: knowledge management perspectives and "bottlenecks"}

The idea that most of the knowledge and experience in an organization belongs not to the organization itself, but to its employees, has received growing recognition in the KM community during the last decade. Human nature recurs throughout knowledge management literature as a serious barrier to full and efficient usage and creation of knowledge in an organization (Thomas et al., 2001; Cabrera et al., 2006). First, it is linked to the tacit component, inherent in the knowledge an individual possesses (Spender, 1993). Second, there are motivational and other individual barriers that may inhibit knowledge-sharing processes from both the knowledge owner's and knowledge recipient's sides (e.g. Husted and Michailova, 2002). Therefore, discussion of the approaches and tools that can help organizations to manage their employees' knowledge has been intensive (e.g. Robertson and Hammersley, 2000; Currie and Kerrin, 2003; Cabrera and Cabrera, 2005).

Nevertheless, KM discourse has been recently criticized for over-focusing on the organizational level of analysis and not paying enough attention to the knowledge-related behavior of individuals (Foss and Felin, 2006). Indeed, there has been much discussion on knowledge sharing (e.g. Argote and Ingram, 2000; Husted and Michailova, 2002; Bock et al., 2005), yet very few sources discuss the question of how knowledge owners obtain something to share. In other words, if a lot of human knowledge is tacit and to a certain extent not even recognized by the owner, what can and should individuals do to extract this knowledge from their own minds, in order to make it available to share with others?

One of the few sources that address this issue is the SECI model of knowledge creation proposed by Nonaka (1991), further developed by Nonaka and Takeuchi (1995). Developing the distinction between tacit and explicit knowledge, they suggested that the creation of new knowledge in organizations can be described with a four-stage spiral model. The second phase of this model, articulation or externalization, involves transformation of the tacit into explicit knowledge. How can this be achieved?

Nonaka and Takeuchi argue that of all four conversion modes, the externalization phase is the most difficult and time-consuming. To help externalization, they recommend the extensive use of metaphors, analogies and models in all discussions in a company, and also to create conditions for the open dialogue of employees with each other and with managers and for the development of communities of practice. These tools can be very useful; however, they are also subject to some limitations.

PAGE 524 | JOURNAL OF KNOWLEDGE MANAGEMENT $\mid$ VOL. 16 NO. 42012 
The key issue is that the tools and methods proposed by Nonaka and Takeuchi for externalization can be labeled as "individual-driven". Indeed, they make an implicit basic assumption about the proactive position of the knowledge owner, who is viewed as inherently willing to make (a lot of) effort to identify, articulate and share his/her knowledge, as well as being capable of completing this task. The literature on human behavior suggests that in order to perform an activity well, an individual needs to have simultaneously motivation, opportunity and the ability to perform (usually referred to as MOA, e.g. Blumberg and Pringle, 1982). The MOA framework applies to the knowledge-related activities and performance of an employee (Kelloway and Barling, 2000; Foss and Minbaeva, 2009). Although it is just one of the concepts used to describe human behavior, the authors propose that MOA might be very useful for analysis of knowledge-related behaviors in organizations, as it incorporates the elements of both individual and organizational levels of analysis and allows discussion of the interplay between them; it counteracts the recent criticism that the $\mathrm{KM}$ literature has been overlooking micro-level issues and inter-level relationships (Foss, 2007).

Based on the MOA model, the authors argue that Nonaka and Takeuchi's vision of the knowledge owners inside an organization is somewhat idealistic. First, the frequent lack of an individual's motivation to share knowledge (sometimes referred to as knowledge hoarding) has been widely acknowledged (e.g. Husted and Michailova, 2002; Bock et al., 2005; Cabrera et al., 2006). Second, organizations have frequently been blamed for not providing sufficient opportunities for knowledge sharing (e.g. Curtis and Leon, 2002; Miles et al., 2009). The notion of "opportunity" in an organizational setting may refer to a wide array of issues that include organizational structure, job design, task requirements, communication channels, provision of ICT, and organizational norms and values; in other words, to organization-level determinants of behavior (e.g. Hendriks, 1999; Jones et al., 2006; Foss et al., 2009). Externalization of one's own tacit knowledge is one of the steps in the knowledge sharing process, and thus is also liable to lack of motivation and opportunity. The third element of the MOA framework, capability, has received less attention in the knowledge management literature. Nevertheless, there is evidence that some individuals are more capable of externalizing and sharing their knowledge than others (Minbaeva and Michailova, 2004; Reinholt et al., 2011). Therefore, it is quite unrealistic to suppose that all knowledge owners in an organization will be sufficiently motivated, capable and have a chance to invest their efforts into transferring their experience into explicit knowledge, and thus the applicability of the individual-driven tools is highly limited. Further, these tools, being individual-driven, mainly consider individual or small group tacit knowledge. Yet tacit knowledge may also exist at the collective level (Spender, 1996). Therefore, there is a challenge to elicit organization-wide tacit knowledge, especially if not all members of the collective have an "ideal" MOA disposition. In addition, the tools proposed by Nonaka and Takeuchi may have some cultural limits to their applicability (e.g. Glisby and Holden, 2003; Andreeva and Ikhilchik, 2011), meaning that they may not be equally efficient in different cultural contexts, organizational, industrial or national.

Based on this discussion, the logical question arises - what other tools might there be that managers can use in order to ensure the best possible flow of knowledge externalization in their companies? The authors suggest that knowledge engineering can inform this, and turn to its accomplishments in the next section.

\section{"The question of what managers can do (if anything) to transform their employees' knowledge into organizational knowledge, or at least to share it as much as possible among various individuals in an organization, remains largely open."}




\section{Knowledge of individuals: insights from knowledge engineering}

\subsection{Bridging knowledge engineering and knowledge management}

Knowledge engineering as a subfield of intelligent system development research area can offer a much richer list of tools and methods to enhance the process of elicitation of knowledge from individual employees and its consequent use in an organization. Indeed, $\mathrm{KE}$ has rapidly developed a range of techniques and tools for these purposes (e.g. Boose, 1989; Adeli, 1994; Milton et al., 2006). These developments have underpinned an emerging methodology that can bridge the gap between the remarkable capacity of the human brain to structure and store knowledge and the knowledge analyst's ability to model this process. Knowledge engineering is still rather new, a multidisciplinary domain that draws upon areas like cognitive science, knowledge elicitation, structuring and formalization.

The initial idea of KE was to build IT-supported expert systems that would store valuable knowledge detached from the individual expert and make it available to many users. However, trying to accomplish this task, knowledge engineers soon discovered that acquiring sufficient high-quality knowledge from individuals to build a robust and useful system was a very time-consuming and expensive activity. Therefore, knowledge elicitation was identified as the bottleneck in building an expert system. This led to knowledge elicitation becoming a major research field within knowledge engineering (Gullen and Bryman, 1988; Diaper, 1989; Gavrilova and Chervinskaya, 1992). The aim of knowledge elicitation was formulated to develop methods and tools that make the arduous task of capturing and validating an expert's knowledge as efficient and effective as possible. At the same time, many knowledge engineering sources stress that experts are frequently important and busy people and their time is costly; hence it is vital that the methods used minimize the time each expert spends off the job taking part in knowledge elicitation sessions (e.g. Morgoev, 1988; Moore and Miles, 1991).

From this discussion one can see that KM and KM identified the same key challenge, though they approached it from different perspectives and even used different vocabulary. KE discourse frequently uses another term - knowledge acquisition. However, in this paper the term "knowledge elicitation" is used, as "knowledge acquisition" has a different meaning in KM discourse (e.g. Cohen and Levinthal, 1990). Thus, it makes sense to bridge the accomplishments of these two disciplines in solving this problem and through this to enrich each other's field. This paper focuses on bringing insights from KE into the KM field. KE discusses the wide range of issues related to knowledge elicitation, structuring and representation; however, in this particular paper the focus is on its ideas in the field of knowledge elicitation.

As a first step in bridging the findings of the two disciplines, a thorough review of the KE literature regarding various knowledge elicitation methods was carried out, by examining relevant papers published in scientific journals. As a first step of this review, the search in titles, abstracts and keywords for "knowledge elicitation" and "knowledge elicitation methods" in international journal databases (ABI Inform Global, EBSCO, Sage and Emerald Management Xtra) and scholar.google resources was performed. The authors did not limit the time period, and thus in some cases turned to the initial publications, dating back to the 1980-90s. However, the biggest group of articles retrieved dates from the mid-1990s to 2011. The first wave of publications to discuss knowledge elicitation methods can be dated approximately from 1985 to 2000 and appeared mainly in journals that deal with knowledge engineering for intelligent system design and development (e.g. Artificial Intelligence Review; Expert Systems; International Journal of Human-Computer Studies; Knowledge-Based Systems; Knowledge Acquisition; Journal of Computer Information Systems). Later (2000-2011), another wave of publications appeared that addressed the topic more from managerial point-of-view (e.g. in journals like Interdisciplinary Journal of Information; Knowledge and Management; Journal of Management Studies; Organization Science; Strategic Management Journal; Knowledge and Process Management; Human Resource Management; Journal of Knowledge Management). 


\section{"Very few sources discuss the question of how knowledge owners obtain something to share."}

The initial search identified over 100 articles. However, since the present analysis focuses on the applicability of various knowledge elicitation techniques, the many papers that mention methods only in theory or deal with them rather generally were left aside. The authors also studied some of the reference lists of the identified articles, and thus were able to identify additional publications. This step also suggested that there was a need to use another keyword for the search, "knowledge acquisition", which frequently replaces "knowledge elicitation". Therefore, an additional search of the same databases was performed. The analysis of reference lists from the both search sets further suggested that there was a need to spread the review to other related streams of research, such as "knowledge capture" and "knowledge extraction".

As the next step in the analysis, the identified literature sources were analyzed through the prism of the twenty years' experience of one of the authors in the application of various knowledge elicitation techniques in a variety of industries and organizational settings. The authors also studied comments from group discussions of practitioners who had taken the "knowledge engineering" course taught by one of the authors over several years in a number of universities. The following section presents the ideas built from these triangulated sources.

\subsection{Knowledge elicitation: roles of an expert and an analyst}

The knowledge engineering literature identifies two distinct roles in the knowledge elicitation process: expert and analyst (Waterman, 2004; Kendal and Creen, 2006). An expert is an individual who possesses valuable knowledge that is of interest to an organization, and thus needs to be elicited. In terms of knowledge management, the majority of employees can be labeled as "experts", as long as they possess some knowledge that is of value to an organization. The second role, the analyst, is a person who is responsible for eliciting knowledge from an expert. An analyst is indeed a key figure from the knowledge engineering perspective, as he/she has special skills and knowledge that enable him/her to elicit knowledge from the expert. An analyst also has a mandate from an organization to spend time and effort on knowledge elicitation, and holds responsibility for the success of this task. Therefore, the analyst acts as an intermediary between an expert and his knowledge, on the one side, and an organization (a knowledge base and/or individual members of the organization) on the other side, the goal being to facilitate knowledge transfer between these two sides.

An interesting difference between KE and KM is that the role of analyst does not exist in the latter. This may be explained by the implicit assumption of KM discussed above about the proactive position of the owner of knowledge. If a knowledgeable employee is ready to spend time on proactive efforts in the elicitation of his/her knowledge (he/she is motivated, appropriately skilled and provided with opportunities), then an intermediary like an analyst is not needed. However, as was argued in section 2, such assumptions about knowledge owners are unrealistic and have serious limitations. Therefore, the authors suggest that introducing the role of analyst may help to overcome some of these limitations, and thus enrich KM practice in organizations by enabling knowledge elicitation and transfer under unfavorable conditions (i.e. when a knowledge expert lacks some of the elements of the MOA framework).

Introducing the role of analyst not only allows compensating for the lack of some of the characteristics of a knowledge owner (expert). It also opens the way to a number of knowledge elicitation methods that cannot be exercised by an expert solely on his/her own and require some type of collaboration with another, motivated and skilled, individual. In other words, while KM usually discusses the techniques that can be used by a proactive knowledge owner (like metaphors, models and analogies, offered by Nonaka and Takeuchi, 
1995), KE considers not only "proactive" but also "reactive" knowledge elicitation techniques that can overcome the MOA deficiencies of an expert. Some of these methods are discussed in the next section.

\subsection{Knowledge elicitation methods: various types}

Since the early 2000s a major interest of knowledge engineering researchers has been the techniques and tools that help knowledge capture, not only for the development of intelligent systems but also for knowledge management practices. This new generation of tools is concerned with knowledge elicitation procedures that facilitate knowledge sharing and reuse (Burge, 1996; Gavrilova and Laird, 2005; Voinov and Gavrilova, 2008). As the palette of techniques is quite extensive, the main question that arises is how to choose an appropriate knowledge elicitation method that would fit a specific situation and its requirements.

Various classifications of methods may serve this purpose. A number of taxonomies have been proposed by knowledge elicitation researchers (e.g. Gavrilova, 1993; Moody et al., 1998). Waterman (2004) divided knowledge elicitation methods into two simple categories: indirect and direct. Hoffman, Burton and Klein (Hoffman et al., 1995) made a deeper overview of techniques and grouped knowledge elicitation methods into three categories: analysis of tasks that the expert performs, various types of interview, and contrived techniques. However, from the perspective of $\mathrm{KM}$ a different taxonomy that highlights the distinct roles of the analyst and the expert and potential variations of these roles would be more useful. The authors propose such a taxonomy in Figure 1. A novel element in their approach is based on the idea of the leading role of the analyst who works as a facilitator and organizer of knowledge elicitation processes. He/she serves as an interface between the knowledge owners and recipients. The authors suggest that this taxonomy of methods can enrich KM practice by introducing the role of an analyst and structuring knowledge elicitation methods according to the different functions an analyst may perform.

This taxonomy descends from several attempts to classify the variety of knowledge elicitation techniques (Gavrilova, 1993; Burge, 1996; Milton, 2008). As introducing the role of the analyst and extracting tacit knowledge are the main focus of our paper, an array of indirect methods (such as data mining and other computer-aided techniques) is excluded from the taxonomy as they do not involve the interaction of an expert and an analyst; similarly, secondary methods (such as repertory grids, rating, visual concept mapping and card sorting) are also excluded as they can only be used after application of the methods presented in Figure 1, at the next stage of knowledge elicitation and structuring (the methods omitted here are well discussed in other publications, e.g. Coffey and Hoffman, 2003; Kwong and Lee, 2009).

Figure 1 Taxonomy of knowledge elicitation techniques

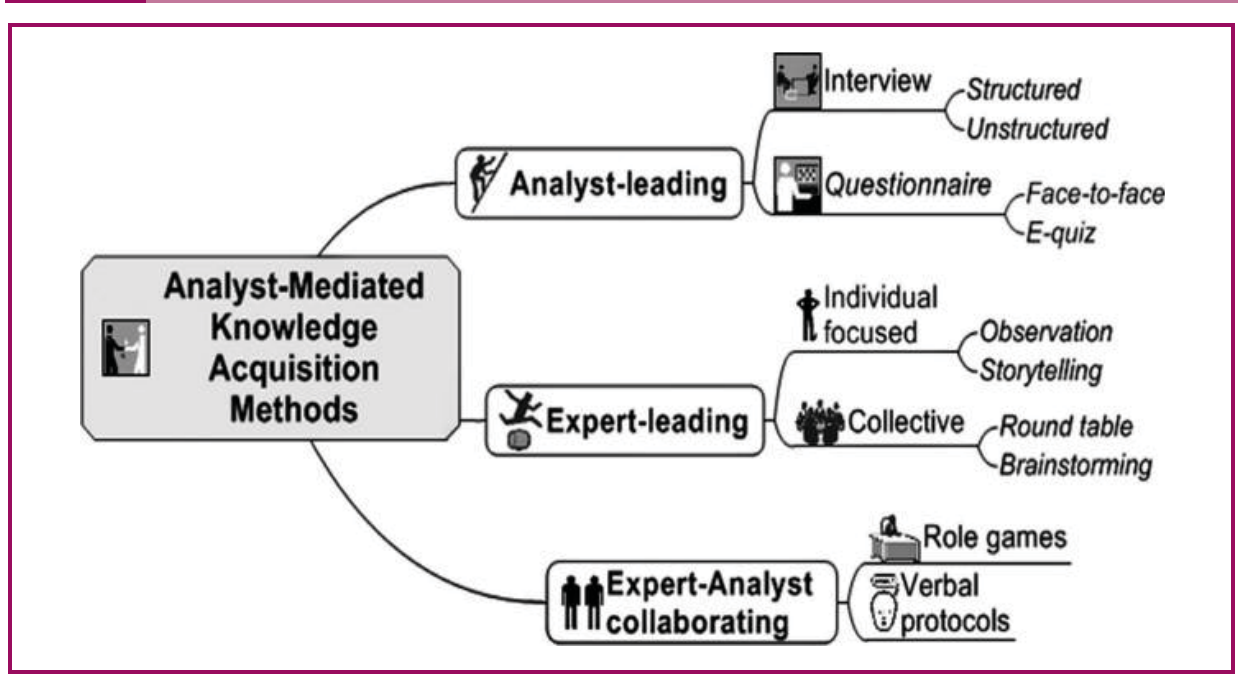

PAGE 528 | JOURNAL OF KNOWLEDGE MANAGEMENT $\mid$ VOL. 16 NO. 42012 


\title{
"It is quite unrealistic to suppose that all knowledge owners in an organization will be sufficiently motivated, capable and have a chance to invest their efforts into transferring their experience into explicit knowledge, and thus the applicability of the individual-driven tools is highly limited."
}

\begin{abstract}
The methods described in the proposed taxonomy range from informal techniques such as "verbal reports" and "observation" through common social science methods such as interviews and questionnaires to more formal techniques used in knowledge-based systems' development in computer science (Cordingley, 1989; EAGLES, 1996). The authors propose to divide these methods into three categories using such criteria as the level of involvement of an expert and an analyst, and type of interaction/collaboration between them.

Two of these three categories can be labeled as "passive" and "active" methods respectively (from the perspective of the level of involvement of an analyst as compared to the efforts of an expert), and the third category implies more or less equal involvement of both parties. By "active" (analyst-leading) methods the authors mean the techniques that require the active position of an analyst, who "pulls" the knowledge from the expert with the help of specially prepared questions. By "passive" (expert-leading) methods the authors mean the techniques that imply that the analyst's interference into the process in which the expert is engaged is very limited; therefore, these methods might be more prone (compared to "active" methods) to problems in case an expert lacks some elements of MOA framework. Observation is a good example of a "passive" method, where the role of the analyst is just to observe/listen and then analyze.
\end{abstract}

In the next paragraphs, the knowledge elicitation methods mentioned in the proposed taxonomy are briefly introduced, along with their advantages and disadvantages.

3.3.1 Analyst-leading methods. Interview is a specific form of communication between the analyst and the expert, where the analyst asks a number of questions prepared in advance in order to gain a better understanding of a specific knowledge area (Matarozzo et al., 1963; Shumilina, 1973; Belnap and Steel, 1976; Belanovsky, 2003; Bradburn et al., 2004; Rollnick et al., 2007; Hashem, 2008). There are different views on interviews in journalism, healthcare, sociology, marketing and other sciences. The interview may have different levels of organization (structured, unstructured, semi-structured) that gives the analyst different levels of freedom. Interview is the most popular technique because of its apparent simplicity of conducting. However, experience shows that best practices in interviewing need years of training and practical fieldwork. The main mistakes are based on the short and superficial stage of preparation to this method of knowledge elicitation (Gavrilova, 1993). Due to their character, interviews are generally aimed at elicitation of explicit knowledge from individuals.

Questionnaire is a highly formalized method, targeted mainly at data collection. Questionnaires do not work at all for elicitation of tacit knowledge, as by their nature they address only already verbalized, formalized knowledge and do not allow for deeper probing.

The analyst formulates a list of questions in advance and presents them to a number of experts. Perhaps the most comprehensive experience of questionnaire application has been accumulated in sociology and psychology (Andersen and Taylor, 2010). There are several general recommendations for composing a questionnaire (e.g. Yadov, 1998). Nowadays, web-based methods of organizing questionnaires have become increasingly popular (e.g. www.surveymonkey.com). Such e-quizzes serve as electronic tools that help to

VOL. 16 NO. 42012 | JOURNAL OF KNOWLEDGE MANAGEMENT | PAGE 529 
simplify data collection and processing but still require very careful professional preparation and financial investment.

3.3.2 Expert-leading methods. Expert-leading methods can be split into individual-focused and collective methods. Individual-focused methods are observation and storytelling (lecturing), while collective methods include round-table and brainstorming.

The observation method implies that the analyst is located in direct proximity to the expert, observing closely his professional activities or their imitation. Before the session, the analyst explains the purpose of the observation to the expert. The analyst should record actions made by the expert, all his/her remarks and comments. A video recording is usually helpful given that the expert has provided consent. The key precondition of this method is the avoidance of any intrusion by the analyst into the work of the expert. For this reason, the method is seen as the only "pure" method, excluding interventions or influence on the cognitive process by the observer. For this reason it may lead to the serendipitous revelation of pieces of tacit knowledge.

Observation of the imitation of a process is also applied in situations where the observation of a real-life process is impossible. The scripts from the observation sessions should be transcribed in detail soon after the session and verified with the expert. There is no possibility of asking for feedback or additional comments from the expert in this method, and this represents both its advantage and its drawback. On the one hand, the process runs uninterrupted so it better represents the expert's activity and reasoning, but, on the other hand, the analyst's interpretation of the observed activity might be incorrect if he/she does not fully understand it.

On the surface, storytelling (lecturing) seems to be a rather simple method. Lecturing is probably one of the oldest forms of knowledge transfer. The art of lecturing has been highly regarded in science and the humanities since ancient times. However, in order to be efficient, lecturing requires not only the expert's ability to prepare and conduct lectures, but also the recipient's ability to listen, transcribe and understand the material (Gibbs, 1989).

On the one hand, the experience of experts differs: they may or may not have the expertise and the talent to lecture. If the expert has lecturing experience, the knowledge transfer in the form of a lecture could represent a concentrated and structured knowledge fragment. On the other hand, potential knowledge recipients may also differ in their capacity to absorb knowledge, and therefore an analyst as mediator might be needed in some situations. Storytelling also allows a significant degree of freedom; however, the topic and objectives of the story should be clearly formulated in advance. When the topic and objectives are provided, an experienced speaker can structure his/her knowledge and refine the logic in advance. The recipient will need to diligently transcribe the lecture and to ask questions for clarification. The method is based wholly on verbalization, so it commonly reveals individual explicit knowledge.

The round-table method prescribes the discussion of a given topic by a number of experts, each given equal rights (Hill, 1980). The number of participants can vary from three to seven. The analyst is required to make the additional effort of both an organizational (e.g. preparation of location, coordination of time, place, etc.) and a psychological kind (e.g. ability to input relevant comments, good memory for names, etc.). When transcribing and analyzing records of round-table discussions, the peer pressure effect, along with

\section{"Being able to choose the best methods for elicitation of specific types of knowledge is important as it contributes to the further development and deployment of knowledge within an organization."}


established relations between participants, should be carefully considered. The method is rather costly so it is used in practice only as additional for the resolution of disputes.

The brainstorming method is aimed at facilitating new ideas, with the prohibition of any critique (Osborn, 1963). It is based on the observation that critique impedes creative thinking, so the essence of brainstorming is to divide the process of idea generation from critical analysis and valuation of the ideas that emerge. A valuation of ideas accumulated during the brainstorming session is usually done by a group of experts who have not participated in the session (Oppenheim, 1986). The method is exciting but not very fruitful from the knowledge elicitation point-of-view, as it mainly helps with elicitation of new ideas and knowledge creation but not with elicitation of the existing thinking patterns and activity routines of the experts.

3.3.3 Expert-analyst collaboration methods. Expert-analyst collaboration methods include role-playing games and verbal protocols. These methods require active positions by both the analyst and the expert.

The role game is a simulation of the professional activity, and implies the participation of several experts. The game is played according to a given scenario; all roles are assigned in advance, and each role has a description and performance valuation matrix (Newstrom and Scannell, 1995). Expert games, like any business games, need an extraordinary amount of professional maturity from the analyst. The game design, scenario and preparation are creative work, but this stage is really time-consuming. However, the result may be outstanding as the game activates the experts' minds and reveals their tacit and implicit decision-making procedures.

The record of a verbal report (protocol) supposes that the expert is asked to both comment on his actions and decisions and explain how those decisions were made, demonstrating the logic used in decision making. While the expert is speaking, his "stream of thought" is carefully recorded, including pauses and exclamations. The method is also called "thinking aloud" or an "improvised lecture" (Burge, 1996). Whether recording devices should be used for this method is debatable as the influence of recording on the expert could be negative. The main drawback of the method is the general difficulty of articulation of one's thinking process. Indeed, experimental psychology has demonstrated that people are not always able to describe their own thinking processes (Eysenck and Keane, 2005). Furthermore, a portion of the knowledge stored in a tacit form is weakly correlated with its verbal description. The author of a frame system theory, Marvin Minsky, states that an individual could explain what he knows merely as an exception, not as a rule (Minsky, 1974). Transcript of protocols should be made by the analyst observing the session. A successful protocol for the procedure of "thinking aloud" is one of the most efficient methods of knowledge extraction, as it allows the expert to freely reveal his thinking process. The attitude towards this self-reflection method among practical analysts differs - while some consider this method as the most desirable and pleasant, other refuse to participate in it as they find simultaneous doing and explaining very challenging, and also threatening to their expert image.

\subsection{Knowledge elicitation methods for different types of knowledge}

In order to navigate through the variety of knowledge elicitation methods, one needs to understand which method best fits the particular problem and situation. This need for a contingency-based approach to the choice of methods has been widely acknowledged in knowledge engineering discourse. For example, Milton et al. (2006) formulate the following key principles of knowledge elicitation:

- there are different types of knowledge;

- there are different types of experts and expertise;

- there are different ways of representing knowledge, which can aid the elicitation, validation and re-use of knowledge;

- there are different ways of using knowledge, so that the elicitation process can be guided by the project aims; and

- therefore, knowledge elicitation methods should be chosen appropriately to fit all contingencies.

VOL. 16 NO. 42012 | JOURNAL OF KNOWLEDGE MANAGEMENT | PAGE 531 
From a knowledge management perspective, the types of knowledge represent the key contingency that could guide the choice of knowledge elicitation method. The distinction between tacit and explicit knowledge is one of the basic principles of KM (e.g. Nonaka and Takeuchi, 1995; Spender, 1996). Although basic, it is especially important for discussion of the knowledge elicitation techniques, as an expert, as a human being, inherently possesses tacit knowledge, and in some fields and situations the extent of the tacit knowledge can be extremely wide. Another characteristic of knowledge that is important for the current discussion is who holds it - individuals or collectives. As section 2 of this paper indicates, KM discourse typically focuses on individually held knowledge. Thus identification of what methods can be used to elicit collective knowledge could be very useful for knowledge management practice.

Table I maps the knowledge elicitation techniques described above on to these types of knowledge. This mapping represents the authors' opinion that emerged from the analysis of the literature, describing the essence of each method and one author's practical experience with application of these methods. It may help practitioners in both KM and KE to choose the appropriate method of knowledge elicitation. There are many other characteristics of knowledge that may be considered for such mapping (e.g. Holsapple, 2004); however, the authors suggest that distinctions of tacit vs explicit and individual vs collective knowledge are basic (Spender, 1996) and thus should be used the first stage of deciding on the choice of methods.

Analysis of this table suggests that more methods are suitable for elicitation of explicit and individual knowledge than for elicitation of tacit and group knowledge. However, it indicates that although they are fewer in number, methods for the elicitation of tacit and group knowledge do exist, suggesting useful implications for practitioners.

The relevance of knowledge assets as fundamental strategic factors in business success has been widely recognized in today's competitive scenario (Barney, 1991). In fact, more and more organizations credit their competitiveness essentially to their knowledge assets and consider knowledge as the differentiating competitive lever in knowledge economy (Nonaka and Takeuchi, 1995). Given this situation, suitable development and deployment of a company's knowledge assets has become a strategic factor for success. From this perspective, being able to choose the best methods for elicitation of specific types of knowledge is important as it contributes to the further development and deployment of knowledge within an organization.

\section{Conclusions}

This paper makes several contributions to the contemporary discourse on managing knowledge in organizations. First, it bridges two different streams of academic research that are usually treated as independent: knowledge management and knowledge engineering. Second, it suggests how KM practitioners (either specially assigned analysts or any organizational members that are interested in eliciting knowledge) can choose the appropriate method for concrete knowledge elicitation, using the taxonomy and the

Table I Comparison of the knowledge elicitation methods

\begin{tabular}{lcc} 
Elicitation methods & Tacit & Exp \\
\hline Interview & $*$ & $*$ \\
Questionnaire & - & $*$ \\
Observation & $* *$ & $*$ \\
Storytelling & $*$ & $*$ \\
Round table & - & $*$ \\
Brainstorming & $* *$ & $*$ \\
Role game & $* *$ & $*$ \\
Verbal protocols & $*$ & $* *$
\end{tabular}

Type of knowledge

Explicit Individual

Group

Notes: The legend used in the table is the following: ${ }^{*}$ May work; ${ }^{* \star} \mid s$ appropriate; ${ }^{\star \star \star} \mid$ Is very suitable, - not applicable 
mapping of methods proposed here. Third, it may serve as a starting point for further investigation of practical KM techniques. Last, but not least, it draws the attention of KM practitioners to the role and qualifications of the knowledge analyst who serves as a mediator between the expert's knowledge and the company's knowledge memory. The role of the analyst has been discussed for decades in KE, but has been unfairly overlooked in KM discourse and practice, and this paper tries to close this gap.

The analysis provided here also enriches the discussion on innovation in the organization. If, as Nonaka and Takeuchi (1995) posit, transformation of tacit into explicit knowledge is one of the cornerstones of knowledge creation, the better an organization is able to elicit tacit knowledge from its employees and share it across organization, the more innovative it can be.

This paper focused on enriching the tools and techniques of $\mathrm{KM}$ with findings from $\mathrm{KE}$. However, the authors suggest that cross-enrichment of these two disciplines could work both ways. For example, discussion of knowledge elicitation methods in KE is dominated by two limitations of the experts: the cost of their time, and the tacitness of their knowledge. Yet, as $\mathrm{KM}$ discourse suggests, the motivation of an expert (as well as his/her inclination to knowledge hoarding) may also influence the outcomes of knowledge elicitation techniques. Therefore, the KE field can benefit from a better understanding of the motivational dispositions of an expert. This is one of the possible future directions for bridging and enriching these two disciplines.

Another future direction for discussion between $\mathrm{KM}$ and $\mathrm{KE}$ refers to the potential for application of other knowledge elicitation methods in the KM context. For example, the proposed classification may be enriched by a group of so-called indirect knowledge elicitation methods that includes computer-based methods (CBM) such as data- and web-mining techniques. Data mining, also known as knowledge discovery, is the practice of automatically searching large stores of data for meaningful patterns (knowledge). With the advances in the process of data collection, the 1990s witnessed an explosion in the growth of data available online. This, coupled with the stellar advances in computing technologies, really improved the image of "data mining". The web is the largest dynamic and online store known today, and web mining refers to the process of extracting knowledge from web pages by exploring their contents, the hyperlinks that connect these pages, or usage patterns of users (Snásel and Kudelka, 2009). This paper did not discuss CBM as these methods do not involve any interaction with an expert and thus fall outside the focus of this paper; however, they might be of interest to knowledge managers. Another area of potential interest is a group of analytical methods that comprises specialized sophisticated techniques that can work with different modes of knowledge. For example, visual-aided techniques help to organize the hierarchical cluster diagram (classification) by card-sorting (Moody et al., 1998) and image-ranking procedures. These techniques can reveal the tacit preferences and priorities in decision making by traditional paper methods that involve the use of stimulus cards and/or pictures/images. These methods are secondary to the ones discussed in this paper as they can be applied only after the initial knowledge has been elicited with the help of the methods described, but they can also enrich the repertoire of the analyst's knowledge elicitation techniques from a knowledge management perspective.

\section{References}

Adeli, H. (1994), Knowledge Engineering, McGraw-Hill Publishing, New York, NY.

Andersen, M.L. and Taylor, H.F. (2010), Sociology: The Essentials, Cengage Learning, Stamford, CT.

Andreeva, T. and Ikhilchik, I. (2011), "Applicability of the SECI model of knowledge creation in Russian cultural context: theoretical analysis", Knowledge and Process Management, Vol. 18 No. 1, pp. 56-66.

Argote, L. and Ingram, P. (2000), "Knowledge transfer: a basis for competitive advantage in firms", Organizational Behavior and Human Decision Processes, Vol. 82 No. 1, pp. 150-69.

Barney, J.B. (1991), "Firm resources and sustained competitive advantage", Journal of Management, Vol. 17 No. 1, pp. 99-120. 
Belanovsky, S.A. (2003), Individual Deep Interview, State Moscow University Press, Moscow, in Russian.

Belnap, N. and Steel, T. (1976), The Logic of Questions and Answers, Yale University Press, New Haven, CT.

Blumberg, M. and Pringle, C. (1982), "The missing opportunity in organizational research: some implications for a theory of work performance", Academy of Management Review, Vol. 7 No. 4, pp. 560-9.

Bock, G.-W., Zmud, R.W., Kim, Y.-G. and Lee, J.-N. (2005), "Behavioral intention formation in knowledge sharing: examining the roles of extrinsic motivators, social-psychological forces, and organizational climate", MIS Quarterly, Vol. 29 No. 1, pp. 87-111.

Boose, J. (1989), "A survey of knowledge acquisition techniques and tools", Knowledge Acquisition, Vol. 1 No. 1, pp. 3-37.

Bradburn, N.M., Sudman, S. and Wansink, B. (2004), Asking Questions: The Definitive Guide to Questionnaire Design - For Market Research, Political Polls, and Social and Health Questionnaires, Jossey Bass Publisher, San Francisco, CA.

Burge, J.E. (1996), "Knowledge elicitation tool classification", Artificial Intelligence Research Group, Worcester Polytechnic Institute, Worcester, MA, available at: http://web.cs.wpi.edu/ jburge/thesis/ kematrix.html (accessed 27 March 2012).

Burton, A.M., Shadbolt, N.R., Rugg, G. and Hedgecock, A.P. (1990), "The efficacy of knowledge elicitation techniques: a comparison across domains and levels of expertise", Knowledge Acquisition, Vol. 2 No. 2, pp. 167-78.

Cabrera, A., Collins, W.C. and Salgado, J.F. (2006), "Determinants of individual engagement in knowledge sharing", International Journal of Human Resource Management, Vol. 17 No. 2, pp. 245-64.

Cabrera, E.F. and Cabrera, A. (2005), "Fostering knowledge sharing through people management practices", International Journal of Human Resource Management, Vol. 16 No. 5, pp. 720-35.

Coffey, J.W. and Hoffman, R.R. (2003), "Knowledge modeling for the preservation of institutional memory", Journal of Knowledge Management, Vol. 7 No. 3, pp. 38-52.

Cohen, W.M. and Levinthal, D.A. (1990), "Absorptive capacity: a new perspective on learning and innovation", Administrative Science Quarterly, Vol. 35 No. 1, pp. 128-52.

Cooke, N.J. (1994), "Varieties of knowledge elicitation techniques", International Journal of Human-Computer Studies, Vol. 41 No. 6, pp. 801-49.

Cordingley, E.S. (1989), "Knowledge acquisition techniques for knowledge-based systems", in Diaper, D. (Ed.), Knowledge Elicitation Principles, Techniques and Applications, Ellis Horwood, Chichester, pp. 89-173.

Currie, G. and Kerrin, M. (2003), "Human resource management and knowledge management: enhancing knowledge sharing in a pharmaceutical company", International Journal of Human Resource Management, Vol. 14 No. 6, pp. 1027-45.

Curtis, R. and Leon, D. (2002), "Supporting knowledge work with physical design", KM Review, Vol. 5 No. 5 , pp. 26-9.

Diaper, D. (Ed.) (1989), Knowledge Elicitation Principles, Techniques and Applications, Ellis Horwood, Chichester.

EAGLES (1996), "EAGLES report", available at: www.issco.unige.ch/en/research/projects/ewg96/ node2.html (accessed 27 March 2012).

Eysenck, M.W. and Keane, M.T. (2005), Cognitive Psychology: A Student's Handbook, Psychology Press, Hove.

Flood, P.C., Turner, T., Ramamoorthy, N. and Pearson, J. (2001), "Causes and consequences of psychological contracts among knowledge workers in the high technology and financial services industries", International Journal of Human Resource Management, Vol. 12 No. 7, pp. 1152-65.

Foss, N. (2007), "The emerging knowledge governance approach: challenges and characteristics", Organization, Vol. 14 No. 1, pp. 29-52. 
Foss, N. and Felin, T. (2006), "Individuals and organizations: thoughts on a micro-foundations project for strategic management", in Ketchen, D.J. and Bergh, D.D. (Eds), Research Methodology in Strategy and Management, Vol. 3, Elsevier, Oxford, pp. 253-88.

Foss, N. and Minbaeva, D. (2009), "Governing knowledge: the strategic human resource management dimension", SWG working paper, 3/2009, Copenhagen Business School, Copenhagen.

Foss, N., Minbaeva, D., Pedersen, T. and Reinholt, M. (2009), "Encouraging knowledge sharing among employees: how job design matters", Human Resource Management, Vol. 48 No. 6, pp. 871-93.

Gavrilova, T. (1993), "Choice of knowledge elicitation technique: the psychological aspect", International Journal of Information Theory and Applications, Vol. 1 No. 8, pp. 20-6.

Gavrilova, T. and Chervinskaya, K. (1992), Knowledge Elicitation and Structuring for Expert Systems Design, Radio and Communication Publishing House, Moscow, in Russian.

Gavrilova, T. and Laird, D. (2005), "Practical design of business enterprise ontologies", in Bramer, M. and Terzyan, V. (Eds), Industrial Applications of Semantic Web, Springer, Heidelberg, pp. 61-81.

Gibbs, G. (1989), Lecturing, Oxford Polytechnic, The Oxford Centre for Staff Development, Oxford.

Glisby, M. and Holden, N. (2003), "Contextual constraints in knowledge management theory: the cultural embeddedness of Nonaka's knowledge-creating company", Knowledge and Process Management, Vol. 10 No. 1, pp. 29-36.

Grant, R.M. (1996), "Toward a knowledge-based theory of the firm", Strategic Management Journal, Vol. 17, pp. 109-22.

Gullen, J. and Bryman, A. (1988), "The knowledge acquisition bottleneck: time for reassessment", Expert Systems, Vol. 5 No. 3, pp. 216-25.

Hashem, A. (2008), Interview Manual, Ramesh Publishing House, Delhi.

Hendriks, P. (1999), "Why share knowledge? The influence of ICT on the motivation for knowledge sharing", Knowledge and Process Management, Vol. 6 No. 2, pp. 91-100.

Hill, L.A. (1980), Techniques of Discussion, Longman, London.

Hoffman, R., Shadbolt, N.R., Burton, A.M. and Klein, G. (1995), "Eliciting knowledge from experts: a methodological analysis", Organizational Behavior and Decision Processes, Vol. 62 No. 2, pp. 129-58.

Holsapple, C.W. (2004), "Knowledge and its attributes", in Holsapple, C. (Ed.), Handbook on Knowledge Management 1: Knowledge Matters, Springer, Berlin and New York, NY, pp. 165-88.

Husted, K. and Michailova, S. (2002), "Diagnosing and fighting knowledge-sharing hostility", Organizational Dynamics, Vol. 31 No. 1, pp. 60-73.

Jones, M.C., Cline, M. and Ryan, S. (2006), "Exploring knowledge sharing in ERP implementation: an organizational culture framework", Decision Support Systems, Vol. 41 No. 2, pp. 411-34.

Kelloway, E. and Barling, J. (2000), "Knowledge work as organizational behavior", International Journal of Management Reviews, Vol. 2 No. 3, pp. 287-304.

Kendal, S. and Creen, M. (2006), An Introduction to Knowledge Engineering, Springer, London.

Kogut, B. and Zander, U. (1992), "Knowledge of the firm, combinative capabilities, and the replication of technology”, Organization Science, Vol. 3 No. 3, pp. 383-97.

Kwong, E. and Lee, W.B. (2009), "Knowledge elicitation in reliability management in the airline industry", Journal of Knowledge Management, Vol. 13 No. 2, pp. 35-48.

Matarozzo, J.D., Weittman, M., Saslow, G. and Weins, A.N. (1963), "Interviewer influence on duration of interview speech", Journal of Verbal Learning and Verbal Behavior, Vol. 1, pp. 451-8.

Miles, R., Miles, G., Snow, C., Blomqvist, K. and Rocha, H. (2009), "The I-form organization", California Management Review, Vol. 51 No. 4, pp. 61-76.

Milton, N. (2008), Knowledge Technologies, Publishing Studies, Polimetrica, Milan.

Milton, N., Clarke, D. and Shadbolt, N. (2006), "Knowledge engineering and psychology: towards a closer relationship", International Journal of Human-Computer Studies, Vol. 64 No. 12, pp. 1214-29. 
Minbaeva, D. and Michailova, S. (2004), "Knowledge transfer and expatriation in multinational corporations: the role of disseminative capacity", Employee Relations, Vol. 26 No. 6, pp. 663-79.

Minsky, M. (1974), A Framework for Representing Knowledge, MIT-AI Laboratory, Cambridge, MA.

Moody, J.W., Blanton, J.E. and Will, R.P. (1998), "Capturing expertise from experts: the need to match knowledge elicitation techniques with expert system types", The Journal of Computer Information Systems, Vol. 1, Winter, pp. 89-95.

Moore, C.J. and Miles, J.C. (1991), "Knowledge elicitation using more than one expert to cover the same domain", Artificial Intelligence Review, Vol. 5 No. 4, pp. 255-71.

Morgoev, V. (1988), "Knowledge elicitation and structuring method: the consulting simulation", Man-Machine Decision Support Systems, VNIISI, Moscow, in Russian.

Newstrom, J. and Scannell, E. (1995), The Big Book of Business Games: Icebreakers, Creativity Exercises and Meeting Energizers, McGraw-Hill, New York, NY.

Nonaka, I. (1991), "The knowledge-creating company", Harvard Business Review, Vol. 69 No. 6, pp. 96-104.

Nonaka, I. and Takeuchi, H. (1995), The Knowledge-creating Company: How Japanese Companies Create the Dynamics of Innovation, Oxford University Press, Oxford.

Oppenheim, A.N. (1986), Creative Thinking and Brainstorming, J. Geoffrey Rawlinson Gower Publishing, Aldershot.

Osborn, A.F. (1963), Applied Imagination: Principles and Procedures of Creative Problem Solving, Charles Scribner's Sons, New York, NY.

Reinholt, M., Pedersen, T. and Foss, N. (2011), "Why a central network position isn't enough: the role of motivation and ability for knowledge sharing in employee networks", Academy of Management Journal, Vol. 54 No. 6, pp. 1277-97.

Robertson, M. and Hammersley, G.O. (2000), "Knowledge management practices within a knowledge-intensive firm: the significance of the people management dimension", Journal of European Industrial Training, Vol. 24 Nos 2-4, pp. 241-54.

Rollnick, S., Miller, W.R. and Butler, C.C. (2007), Motivational Interviewing in Health Care: Helping Patients Change Behavior, The Guilford Press, New York, NY.

Shumilina, T.V. (1973), Interview in Journalism, Moscow State University Press, Moscow, in Russian.

Snásel, V. and Kudelka, M. (2009), "Web content mining focused on named objects", Proceedings of the First International Conference on Intelligent Human Computer Interaction IHCl, pp. 37-58.

Spender, J.C. (1993), "Competitive advantage from tacit knowledge? Unpacking the concept and its strategic implications", Academy of Management Proceedings, pp. 37-41.

Spender, J.C. (1996), "Making knowledge the basis of a dynamic theory of the firm", Strategic Management Journal, Vol. 17, pp. 45-62.

Teece, D.J. (2004), "Knowledge and competence as strategic assets", in Holsapple, C. (Ed.), Handbook on Knowledge Management 1: Knowledge Matters, Springer, Berlin and New York, NY, pp. $129-52$.

Thomas, J.C., Kellogg, W.A. and Erickson, T. (2001), "The knowledge management puzzle: human and social factors in knowledge management”, IBM Systems Journal, Vol. 40 No. 4, pp. 863-84.

Tsoukas, H. and Vladimirou, E. (2001), "What is organizational knowledge?', Journal of Management Studies, Vol. 38 No. 7, pp. 973-93.

Voinov, A. and Gavrilova, T. (2008), "Cognitive reengineering of expert's knowledge by the implicit semantics elicitation", Proceedings of 16th International Conference on Knowledge Engineering and Knowledge Management (EKAW 2008), Knowledge Patterns, Catania, pp. 69-71.

Waterman, D. (2004), A Guide to Expert Systems, Pearson Education, London.

Yadov, V.A. (1998), Strategy of Sociology Study, Dobrosvet, Moscow, in Russian. 


\section{About the authors}

Tatiana Gavrilova is a Head of Information Technologies in the Management Department at St Petersburg University Graduate School of Management, Russia. She is a Head of St Petersburg Division of Russian Association for Artificial Intelligence (RAAI). Dr Gavrilova has more than 100 publications (43 in English) in the field of knowledge engineering and knowledge management; she is the author of five books (in Russian). Dr Gavrilova has lecturing experience in English at the universities of Finland, Italy, Poland and Estonia. In 1998 and 2004 she has been Fulbright Visiting Senior Research Scholar and Lecturer in Penn State University and University of Pittsburgh (USA). From 2000 she has been an IT consultant in one of St Petersburg's consulting companies. Tatiana Gavrilova is the corresponding author and can be contacted at: gavrilova@gsom.pu.ru

Tatiana Andreeva is an Associate Professor at the Organizational Behavior and Human Resource Management Department, St Petersburg University Graduate School of Management, Russia. Her teaching and research are focused on knowledge management, change management, and cross-cultural issues in management. Her current research interests include strategic knowledge management and micro-foundations of knowledge management. She has authored and co-authored several academic articles, papers, and book chapters related to these issues. Prior to joining academia, she worked in management consulting in the area of strategy, organizational development and knowledge management.

To purchase reprints of this article please e-mail: reprints@emeraldinsight.com Or visit our web site for further details: www.emeraldinsight.com/reprints 
This article has been cited by:

1. Jessica Y T Yip, Rongbin W B Lee. 2016. Knowledge elicitation practices for organizational development intervention. Knowledge Management Research \& Practice . [CrossRef]

2. Graham P. Martin, Lorna McKee, Mary Dixon-Woods. 2015. Beyond metrics? Utilizing 'soft intelligence' for healthcare quality and safety. Social Science \& Medicine 142, 19-26. [CrossRef]

3. Jessica Y.T. Yip, Rongbin W.B. Lee, Eric Tsui. 2015. Examining knowledge audit for structured and unstructured business processes: a comparative study in two Hong Kong companies. Journal of Knowledge Management 19:3, 514-529. [Abstract] [Full Text] [PDF]

4. Alexeis Garcia-Perez, Siraj A Shaikh, Harsha K. Kalutarage, Mahsa Jahantab. 2015. Towards a knowledge-based approach for effective decision-making in railway safety. Journal of Knowledge Management 19:3, 641-659. [Abstract] [Full Text] [PDF]

5. Tracy X P Zou, W B Lee. 2014. Eliciting and mapping tacit knowledge on teamwork success of Six Sigma teams. Knowledge Management Research \& Practice . [CrossRef]

6. Tatiana Gavrilova, Margarita Gladkova. 2014. Big Data Structuring: The Role of Visual Models and Ontologies. Procedia Computer Science 31, 336-343. [CrossRef]

7. Susanne Durst, Jessica Yip, Rongbin W. B. LeeSME Succession Planning and Knowledge Loss Assessment 282-298. [CrossRef] 